\title{
Rendimento da Atriplex nummularia irrigada com efluentes da criação de tilápia em rejeito da dessalinização de água ${ }^{1}$
}

\author{
Everaldo R. Porto ${ }^{2}$, Miriam C. C. de Amorim ${ }^{3}$, Maria T. Dutra ${ }^{4}$, Renata V. Paulino ${ }^{5}$, Luiza T. de L. Brito ${ }^{2} \&$ Ana N. B. Matos ${ }^{2}$
}

\section{RESUMO}

Com o objetivo de avaliar a influência no rendimento da erva-sal (Atriplex nummularia) e em algumas características do solo, quando irrigada com efluentes da criação de tilápia (Oreochromis sp.) em rejeito da dessalinização de água salobra no semi-árido brasileiro, a erva-sal foi irrigada durante um ano com quatro volumes de efluentes na Embrapa SemiÁrido, em Petrolina, PE, cuja salinidade média foi, de 8,29 dS $\mathrm{m}^{-1}$. O delineamento experimental foi de blocos ao acaso com quatro tratamentos e três repetições. Os tratamentos T1, T2, T3 e T4 correspondem, respectivamente, aos volumes de efluentes aplicados semanalmente, de 75, 150, 225 e $300 \mathrm{~L} \mathrm{planta}^{-1}$. A salinidade média do solo na profundidade 0 - $90 \mathrm{~cm}$ foi de $0,40 \mathrm{dS} \mathrm{m}^{-1}$, antes de serem iniciadas as irrigações. Após a colheita, respectivamente para os tratamentos T1, T2, T3 e T4, as salinidades médias dos perfis de solo na mesma profundidade $(0-90 \mathrm{~cm})$ foram de 8,02, 6,09, 4,97 e 4,60 dS $\mathrm{m}^{-1}$ e os rendimentos de matéria seca da erva-sal, de 9,75, 12,26, 14,49 e 13,81 t ha ${ }^{-1}$. O maior rendimento de matéria seca por litro de efluente aplicado foi para o tratamento $\mathrm{T} 1$, com 4,84 g L-1 que apresentou, também, a meIhor relação entrada/saída de sal, removendo $13,84 \%$ do total de sal incorporado ao solo.

Palavras-chave: erva-sal, salinidade, irrigação

\section{Behavior of saltbush (Atriplex nummularia) irrigated with effluents from tilapia raised in brackish water}

\begin{abstract}
Looking forward to reduce environmental impacts resulting from desalinization of brackish water in the region of the crystalline rocks in the semi-arid Brazil, saltbush (Atriplex nummularia) was cultivated during one year with four different volumes of aquiculture effluent generated from an intensive tilapia (Oreochromis sp) raising system, with a mean salinity of $8.29 \mathrm{dS} \mathrm{m}^{-1}$, in an experimental field of Embrapa Semi-Arid. A completely randomized block design, with four treatments and three replications was used. The treatments $\mathrm{T} 1, \mathrm{~T} 2, \mathrm{~T} 3$ and T4 corresponded, respectively, to the weekly volumes of irrigation of $75,150,225$ and $300 \mathrm{~L}$ of water per plant. The mean soil salinity of the cropped area, in the depth of 0 $90 \mathrm{~cm}$, was $0.40 \mathrm{dS} \mathrm{m}^{-1}$ before starting the irrigation process. After the plants were harvested, the mean soil salinity in the profiles was 8.02, 6.09, 4.97, and $4.60 \mathrm{dS} \mathrm{m}^{-1}$ for treatments $\mathrm{T} 1, \mathrm{~T} 2, \mathrm{~T} 3$ and $\mathrm{T} 4$, respectively. The saltbush dry matter yields were $9.75,12.26,14.49$ and $13.81 \mathrm{t} \mathrm{ha}^{-1}$ for treatments $\mathrm{T} 1, \mathrm{~T} 2, \mathrm{~T} 3$ and $\mathrm{T} 4$, respectively. The highest dry matter production per unit of applied water was for treatment $\mathrm{T} 1$, which yielded $4.84 \mathrm{~g} \mathrm{~L}^{-1}$ of effluent. The same treatment had the best relation input/output of salt by the saltbush, removing $13.84 \%$ of the total salt incorporated into the soil.
\end{abstract}

Key words: saltbush, salinity, irrigation

1 Trabalho financiado pelo CNPq/CT-HIDRO, Processo 503.229/2003-2.

2 Embrapa Semi-Árido, BR 428, km 152 - Zona Rural, CP 23. CEP 56302 - 970, Petrolina, PE. Fone: (87) 3862-1711. E-mail: erporto@cpatsa.embrapa.br; luizatlb@cpatsa.embrapa.br; ananeryb@yahoo.com.br

${ }^{3}$ Companhia Pernambucana de Saneamento, Av. 31 de Março s/n, Centro. CEP 56300 -000 Petrolina, PE. Fone: (87) 3861-1611. E-mail: miriamcleide@ig.com.br. ${ }^{4}$ CEFET, BR 407, km 08, Jardim São Paulo. CEP 56314-520 Petrolina, PE. Fone: (87) 3863-2330. E-mail: tdutra@yahoo.com.br

${ }_{5}^{5}$ Fundação Banco do Brasil, Rua Ademar de Andrade Silva 125, Cohab Massangano. CEP-56310-640 Petrolina, PE. Fone: (87) 3867-0863.

E-mail: valerenata@yahoo.com.br. 


\section{INTRODUÇÃO}

As águas subterrâneas do semi-árido brasileiro constituem o manancial mais econômico e flexível para atendimento seguro da população, sendo elevado o número de poços tubulares instalados. Devido à peculiaridade geológica de rochas cristalinas, em geral as águas desses poços possuem sais dissolvidos pela intemperização das rochas, inviabilizando seu uso para consumo humano e até mesmo animal. No entanto, a tecnologia da dessalinização permite a viabilização do manancial disponível, pela eficiência no fornecimento de água de boa qualidade, sendo notória a sua expansão.

A crescente expansão da dessalinização poderá trazer impactos ambientais severos devido aos seus subprodutos ou efluentes, isto é, águas com elevados teores de sais que são, na maioria das vezes, despejados no solo indiscriminadamente, conforme indicam estudos de Amorim et al. (1997). A irrigação de plantas halófitas com efluente salino proveniente da exploração de atividades aqüícolas tem sido sugerida como alternativa de redução dos impactos negativos causados pelo não acondicionamento desse subproduto da dessalinização (Miyamoto et al., 1996; Glenn et al., 1998; Brown et al., 1999; Montenegro et al., 2000; Porto et al., 2001).

Halófitas são plantas com habilidade de suportar não apenas altos níveis de salinidade do complexo solo-água mas, também, de acumular significativas quantidades de sais em seus tecidos (Miyamoto et al., 1994). Dentre as halófitas, a erva-sal (Atriplex nummularia) é uma das mais importantes, pelo fato de possuir mecanismos especializados de acumulação de sais no seu interior e de sua eliminação através das folhas. Na realidade, a planta age desta maneira não com o objetivo de se tornar especializada na absorção de sais mas, sim, como forma de se ajustar ao ambiente quando este é de elevada salinidade.

Mesmo a literatura evidenciando a erva-sal como um dessalinizador biológico do solo (Glenn et al., 1998; O’Leary, 1986; Porto et al., 2001), a sua efetividade é maior quando cultivada com volumes menores de efluentes (Brown et al., 1999). Por outro lado, a predominância dos problemas de salinização das áreas irrigadas tem origem no uso excessivo de água na irrigação (Rhoades, 1998), mas é também importante reconhecer a taxa de lixiviação como recomendação de boas práticas na agricultura irrigada; todavia, em se tratando de água salobra, a incorporação de sal no perfil do solo é diretamente relacionada ao volume de água aplicado na irrigação.

O presente estudo tem como objetivo avaliar a influência no rendimento da erva-sal e em algumas características do solo, quando irrigada com quatro volumes de efluentes da criação de tilápia rosa (Oriochromis sp.) em rejeitos da dessalinização de água salobra.

\section{MATERIAL E MÉTODOS}

O experimento foi implantado no Campo Experimental da Caatinga da Embrapa Semi-Árido, em Petrolina, PE onde foram observados os efeitos de quatro volumes de água de irrigação: $\mathrm{T} 1=75 ; \mathrm{T} 2=150 ; \mathrm{T} 3=225$ e T4 = 300 L semana $^{-1}$ planta $^{-1}$, aplicados de uma só vez. O solo da área experimental foi classificado como Argissolo amarelo eutrófico abruptico plíntico. (EMBRAPA, 1999)

Mudas de erva-sal com 30 dias de idade, originárias de uma mesma planta por propagação vegetativa, foram transplantadas para covas de $0,4 \times 0,4 \times 0,4 \mathrm{~m}$, em espaçamento entre covas de 4,0 x 4,0 m, no delineamento experimental de blocos ao acaso, com 16 plantas em cada parcela, cada bloco contendo quatro tratamentos, repetidos três vezes, totalizando 12 parcelas. A área total do experimento foi de $3.072 \mathrm{~m}^{2}$. O sistema de irrigação usado foi o de sulcos e a água aplicada o efluente da criação de tilápia rosa (Oriochromis sp.), cultivada em tanque com o rejeito da dessalinização de água salobra de poço tubular do cristalino. A densidade de povoamento da tilápia no tanque foi de 4 alevinos $\mathrm{m}^{-3}$ e o ciclo do peixe, de seis meses. A borda de cada sulco, com área molhada de $20 \mathrm{~m}^{2}$, continha quatro plantas. O transplante das mudas foi realizado em 16/6/2000 em covas que receberam $5 \mathrm{~L}$ de esterco de caprino e $150 \mathrm{~g}$ de superfosfato simples, cuja colheita ocorreu em 25/6/2001. Durante este período, foram cultivados dois ciclos de tilápia e realizadas 43 irrigações, enquanto, seguindo a metodologia do Laboratório de Solos da Embrapa (Claessen, 1997), o monitoramento da salinidade do efluente e da salinidade do solo foi realizado semanalmente antes de cada irrigação determinando-se a condutividade elétrica ( $\mathrm{dS} \mathrm{m}^{-1}$ ), como também foram determinados o pH e a umidade (\%) do solo. As amostras de efluente foram coletadas e analisadas in loco para determinação de $\mathrm{pH}$ e condutividade elétrica $\left(\mathrm{dS} \mathrm{m}^{-1}\right)$. As amostras de solo foram coletadas antes da aplicação do respectivo volume de água em cada tratamento, nos dias em que eram feitas as irrigações, tomadas a intervalos de $0-30 \mathrm{~cm}, 30$ - 60 cm, 60 - 90 cm, e a uma distância de 0,20 m da planta. Os dados de rendimento total por planta por tratamento foram analisados estatisticamente, avaliados com o teste de Duncan, e submetidos a análises de regressão. Em ambos os procedimentos, usaram-se módulos do SAS (1989). Foram feitas as estimativas de matéria seca (MS), seguindo técnicas descritas por Silva \& Queiroz (1990), além das estimativas dos quantitativos de sais acumulados nos tecidos da erva-sal, por meio da determinação de cinzas totais. Na colheita, retirou-se todo o material vegetal com altura igual ou superior a $50 \mathrm{~cm}$ a partir da superfície do solo, que foi classificado como lenha, caule grosso, caule fino e folha. Como lenha, considerou-se todo o material lenhoso com diâmetro igual ou superior a $10 \mathrm{~mm}$; como caule grosso, o material com diâmetro entre 8 e $10 \mathrm{~mm}$ e, como caule fino, o material com diâmetro inferior a $8 \mathrm{~mm}$. Por fim, foi denominado material forrageiro o conjunto de caules e folha.

\section{RESULTADOS E DISCUSSÃO}

Na Tabela 1 apresentam-se os resultados das análises físicas do solo na área experimental, antes do plantio da ervasal. O solo foi classificado como de textura média/argilosa; 
Tabela 1. Características físicas para diferentes camadas de solo da área de plantio

\begin{tabular}{lcccc}
\hline \multirow{2}{*}{ Características Físicas } & \multicolumn{4}{c}{ Profundidades (cm) } \\
\cline { 2 - 5 } & $\mathbf{0 ~ - ~ 3 0}$ & $\mathbf{3 0 - 6 0}$ & $\mathbf{6 0 - 9 0}$ & Média \\
Areia (\%) & 76 & 63 & 57 & \\
Silte (\%) & 14 & 14 & 17 & \\
Argila (\%) & 10 & 23 & 26 & \\
pH $\left(\mathrm{H}_{2} 0\right.$ - 1:2,5) & 6,1 & 5,8 & 5,2 & 5,70 \\
Condutividade Elétrica $\left(\mathrm{dSm}^{-1}\right)$ & 0,24 & 0,37 & 0,6 & 0,40 \\
Densidade real $\left(\mathrm{gcm}^{-3}\right)$ & 2,58 & 2,63 & 2,61 & \\
Densidade aparente $\left(\mathrm{gcm}^{-3}\right)$ & 1,37 & 1,35 & 1,39 & \\
Capacidade de campo $(\%)$ & 8,49 & 14,35 & 20,86 & 14,56 \\
Ponto de murcha (\%) & 4,38 & 8,82 & 12,19 & 8,46 \\
\hline
\end{tabular}

o pH médio do perfil, de 5,7, é considerado típico para este tipo de solo e a condutividade elétrica do extrato de saturação do perfil do solo foi de $0,40 \mathrm{dS} \mathrm{m} \mathrm{m}^{-1}$ permitindo classificar-se o solo como não salino. A capacidade de retenção de umidade disponível média do perfil, determinada com base em massa, é de 6,1\%.

A Figura 1 apresenta a média diária mensal da condutividade elétrica do efluente durante todo o ciclo de cultivo. Para o período de cultivo, a média geral foi de $8,29 \mathrm{dS} \mathrm{m} \mathrm{m}^{-1}$, podendo o solo ser classificado como salino, para fins de irrigação.

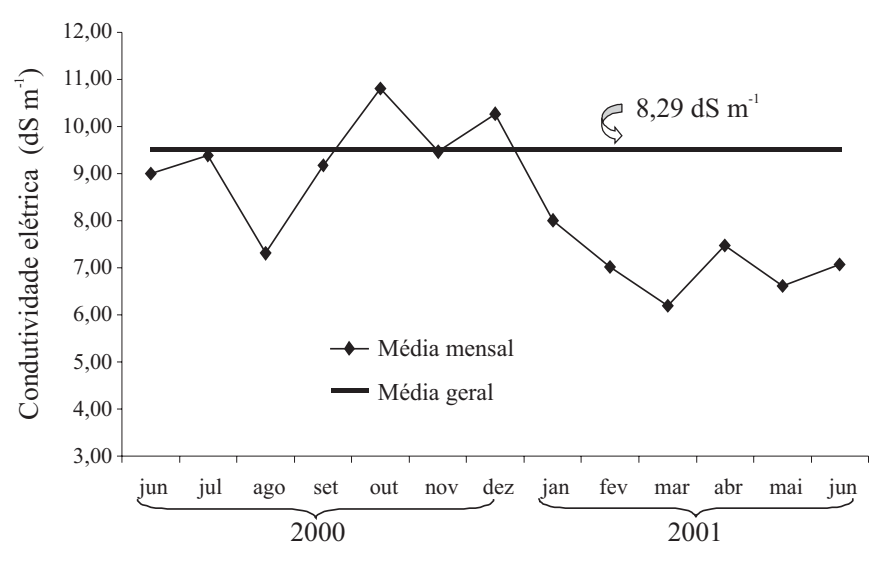

Figura 1. Condutividade elétrica do efluente da criação de tilápia durante o período de cultivo da erva-sal

A Tabela 2 apresenta os dados de $\mathrm{pH}$ e de condutividade elétrica para as três camadas do perfil do solo, de acor- do com os tratamentos. Observa-se um ligeiro aumento nos valores de $\mathrm{pH}$ médios dos perfis, à medida que o volume de água foi maior. Quanto à condutividade elétrica, os aumentos foram significativos e inversamente proporcionais aos aumentos dos volumes de água, mas ainda muito aquém dos limites para comprometimento da produção da erva-sal, que é de 31,25 dS m-1 (20 g L $\left.\mathrm{g}^{-1}\right)$, conforme resultados conseguidos por Miyamoto et al. (1996). É importante observar que, já para o primeiro ano, a condutividade elétrica média do perfil do tratamento T1 aproxima-se da condutividade média do efluente.

Em função de decorrido apenas 1 ano, as maiores concentrações ocorreram nas camadas superiores para todos os tratamentos; todavia, fica evidenciada a possibilidade de ter havido lixiviação no tratamento T4, em virtude de um desvio menor entre os valores da condutividade elétrica das três camadas do perfil, pelo fato do excedente de água da primeira camada do perfil $(0-30 \mathrm{~cm})$ haver transportado parte dos sais para as camadas subseqüentes, o que não se verificou com os tratamentos irrigados com menores volumes que o T4; pelo contrário, por meio do processo de evapotranspiração a solução do solo se foi tornando concentrada no decorrer do período para os tratamentos que receberam menores volumes de água.

O monitoramento semanal da condutividade elétrica do extrato de saturação do solo é apresentado na Figura 2, em que os valores representam médias semanais das três camadas do perfil. Os valores para todos os tratamentos acompanharam a mesma tendência, apresentando valores mais altos na última semana de setembro e na primeira de outubro, quando as taxas de evaporação foram mais altas no tanque classe "A". Como era de se esperar, o tratamento T1 foi o que atingiu os mais altos níveis de salinidade, chegando a um valor médio de 13,43 dS m-1, evidenciando a concentração dos sais em função do reduzido teor de umidade no solo na camada de $0-30 \mathrm{~cm}$. A condutividade elétrica dessa camada para este tratamento, chegou a $17,67 \mathrm{dS} \mathrm{m}^{-1}$.

A Figura 3 apresenta os teores de umidade para os quatro tratamentos. O comportamento dos gráficos obedece a uma tendência dos maiores e menores valores de umidade estarem de acordo com os volumes de água fornecidos para cada tratamento. Considerando-se o conjunto de dados, observa-se que do total das 43 amostragens para cada um dos tratamentos T1, T2, T3 e T4, os valores médios do teor de umidade do perfil foram, respectivamente, 12, 8, 4 e 5 vezes inferiores à média do teor de umidade do ponto de murcha do perfil, que é de 8,46\%. Moore et al. (1972)

Tabela 2. Resultados das análises de solo para as três camadas dos perfis, após um ciclo da erva-sal

\begin{tabular}{|c|c|c|c|c|c|c|c|c|}
\hline \multirow{3}{*}{ Tratamento } & \multicolumn{8}{|c|}{ Profundidade do Solo $(\mathrm{cm})$} \\
\hline & \multicolumn{4}{|c|}{$\mathrm{pH}$} & \multicolumn{4}{|c|}{ CE $250\left(\mathrm{dSm}^{-1}\right)$} \\
\hline & $0-30$ & $30-60$ & $60-90$ & Média do Perfil & $0-30$ & $30-60$ & $60-90$ & Média do Perfil \\
\hline $\mathrm{T} 1$ & 5,20 & 5,80 & 6,10 & 5,70 & 10,69 & 5,96 & 5,96 & 8,02 \\
\hline T2 & 6,00 & 6,30 & 6,10 & 6,13 & 8,73 & 4,99 & 4,99 & 6,09 \\
\hline T3 & 6,40 & 6,90 & 5,70 & 6,33 & 6,54 & 3,63 & 3,63 & 4,97 \\
\hline T4 & 6,90 & 7,00 & 6,00 & 6,63 & 5,22 & 4,71 & 4,71 & 4,60 \\
\hline
\end{tabular}




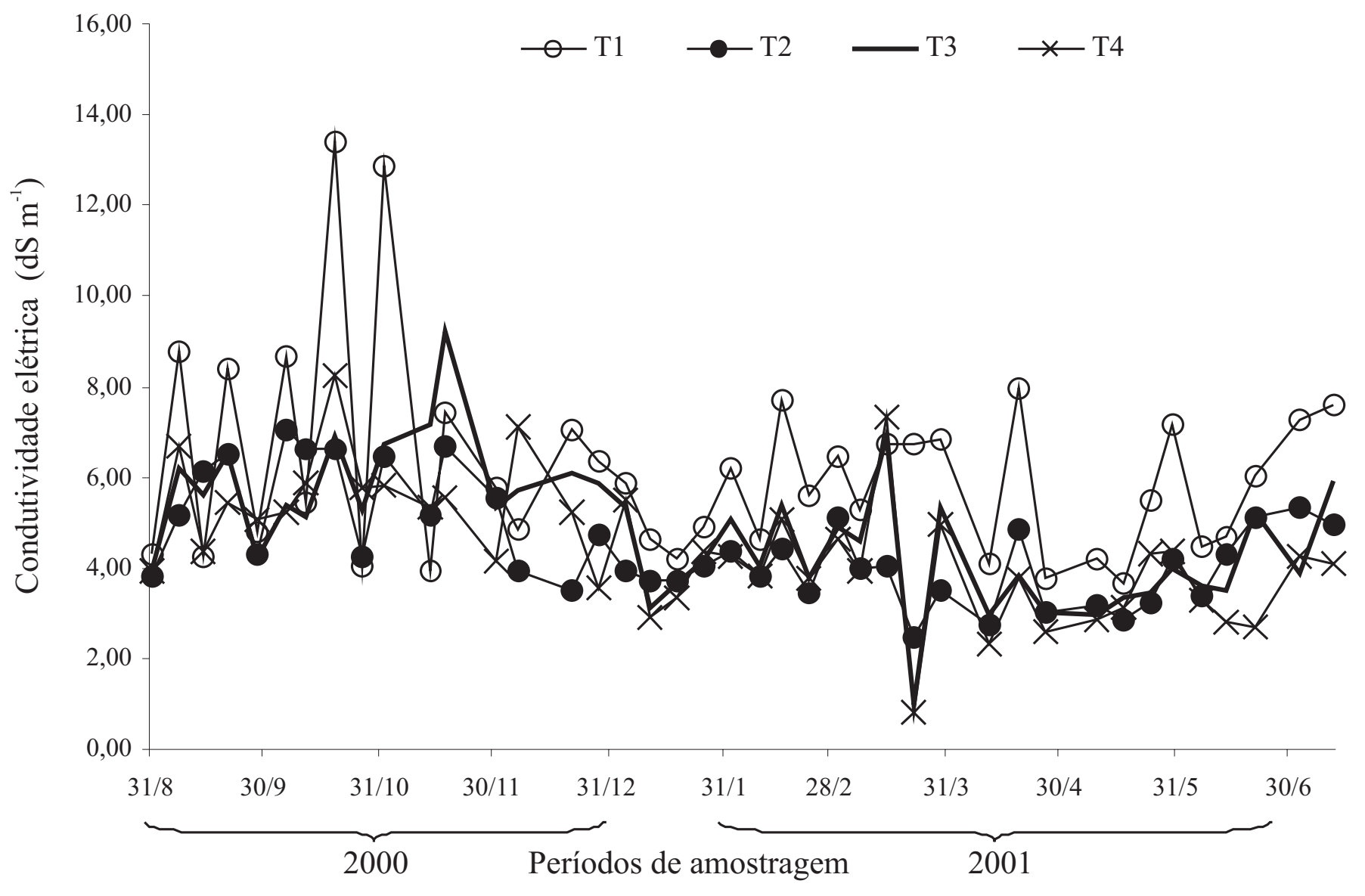

Figura 2. Variação da condutividade elétrica média do extrato de saturação das três camadas do perfil do solo, de acordo com os tratamentos

encontraram que a atriplex continua transpirando, mesmo com teores de umidade no solo inferiores aos do ponto de murcha. É importante observar que o diferencial de umidade entre os tratamentos T3 e T4, durante todo o ciclo de cultivo, é desprezível.

A Tabela 3 mostra o peso médio de matéria fresca por planta, para os diferentes tratamentos. Cada repetição representa a média de quatro plantas colhidas. De acordo com a análise estatística, existem diferenças significativas em nível de 5\%, comprovadas pelo teste de Duncan. O coeficiente de variação foi de $8,16 \%$ e o $\mathrm{R}^{2}$ igual a 0,93 .

Tem-se na Figura 4 a análise de regressão, correlacionando os rendimentos totais por planta e os volumes de água aplicados nas irrigações. De acordo com esta informação, o melhor rendimento físico foi conseguido com o

Tabela 3. Peso total de matéria fresca da parte colhida, por planta, de acordo com os tratamentos e repetições

\begin{tabular}{ccccc}
\hline Tratamento & \multicolumn{4}{c}{ Peso total (kg planta ${ }^{-1}$ ) } \\
& $\mathbf{1}$ & $\mathbf{2}$ & $\mathbf{3}$ & Média $^{*}$ \\
T1 & 50,4 & 47,3 & 46,6 & $48,1 \mathrm{~A}$ \\
T2 & 68,0 & 56,0 & 62,3 & $62,1 \mathrm{~B}$ \\
T3 & 96,0 & 72,0 & 83,9 & $84,0 \mathrm{C}$ \\
T4 & 81,0 & 75,4 & 67,8 & $74,7 \mathrm{C}$ \\
\hline
\end{tabular}

* Médias seguidas da mesma letra não diferem significativamente tratamento T3, ou seja, irrigando-se a erva-sal com o volume de $225 \mathrm{~L}$ semana $^{-1}$ planta $^{-1}$, o que equivale a uma lâmina de água aplicada de $45 \mathrm{~mm}$ semana $^{-1}$ planta $^{-1}$; este valor é próximo ao da taxa de evaporação média semanal, medida na área experimental, com tanque classe "A", isto é tanques cilíndricos de metal e padronizados para medição da evaporação potencial em estações meteorológicas, que foi de 46,9 mm; por outro lado e mesmo havendo diferença de rendimento entre os tratamentos T3 e T4, estatisticamente ela não é significativa, indicando tendência da oferta de água para o rendimento máximo de biomassa ser próximo da evaporação potencial medida pelo tanque classe " $\mathrm{A}$ ".

Considerando-se um estande de 625 plantas ha-1 e a proporcionalidade de cada parte colhida da planta em relação ao total, a Tabela 4 apresenta os resultados das matéria seca para cada tratamento.

Do ponto de vista de produção de forragem, esses resultados podem ser considerados bons, visto que os rendimentos em matéria seca são bem superiores ao conseguido com a leucena (Leucaena leucocephala), que é de 3,0 $\mathrm{t} \mathrm{ha}^{-1} \mathrm{corte}^{-1}$ (Oliveira, 2000) podendo ser comparado ao da alfafa irrigada com água de qualidade superior (O’Leary et al., 1985).

Por outro lado, é conveniente ressaltar que uma das limitações do potencial de água subterrânea no cristalino é a baixa vazão média dos poços. Nesta perspectiva foi calculado, para cada tratamento, o rendimento da erva-sal por litro 

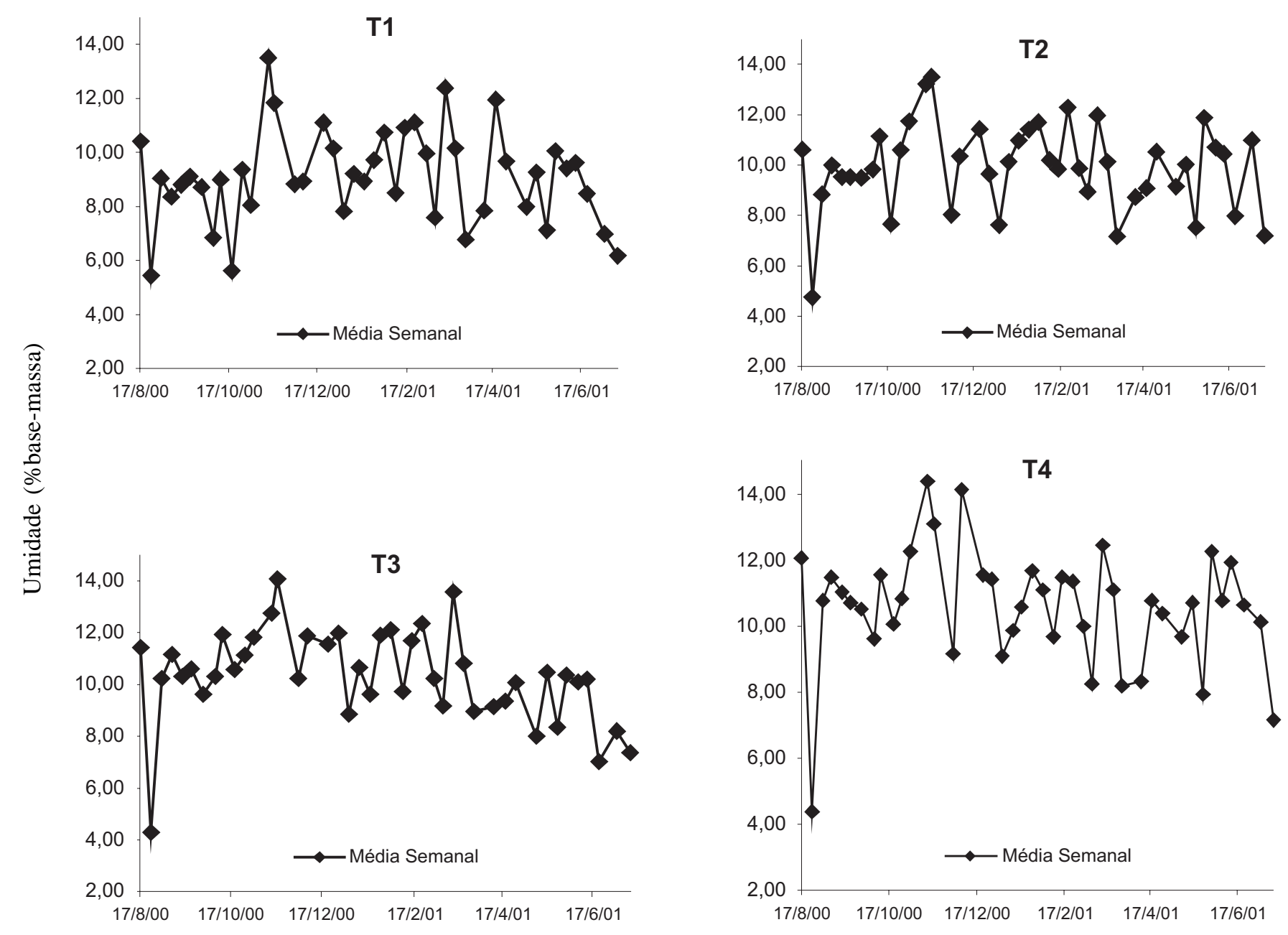

Data da amostragem

Figura 3. Variação dos teores de umidade média das três camadas do perfil de solo, de acordo com os tratamentos

de água aplicado. O tratamento T1 foi o que mais produziu biomassa por unidade de água, atingindo 4,84 g de matéria seca $\mathrm{L}^{-1}$ de efluente. O tratamento T4 produziu apenas $1,71 \mathrm{~g} \mathrm{~L}^{-1}$. Miyamoto (1993) e Miyamoto et al. (1994), encontraram rendimentos de atriplex que variaram entre 1,4 e $2,6 \mathrm{~g} \mathrm{~L}^{-1}$.

Os dados da Tabela 5 apresentam a estimativa da quantidade de cinza existente nas diferentes partes da planta, de acordo com os tratamentos. Os totais de cinza variaram entre 15,0 e 16,0 g $100 \mathrm{~g}^{-1}$ de matéria seca não ha- vendo, portanto, diferenças significativas entre os tratamentos, sendo a maior concentração encontrada na folha que, por sua vez, é o componente mais expressivo do material forrageiro. De acordo com Miyamoto et al. (1994), o limite do teor de sal em ração para caprinos e ovinos é de $10 \mathrm{~g}$ de sais por 100 de matéria seca, sendo esta é uma das limitações do uso da erva-sal como forrageira. Portanto, a recomendação é misturá-la a outros alimentos na formação da dieta diluindo-se desta forma, a concentração de sais na ração.

Tabela 4. Estimativa dos rendimentos totais e de partes da planta de matéria seca ( $\left.\mathrm{kg} \mathrm{ha}^{-1}\right)$, de acordo com os tratamentos

\begin{tabular}{|c|c|c|c|c|c|c|}
\hline \multirow{3}{*}{ Tratamento } & \multicolumn{6}{|c|}{ Parte Colhida } \\
\hline & \multirow{2}{*}{ Total } & \multirow{2}{*}{ Lenha } & \multirow{2}{*}{ Caule Grosso } & \multicolumn{3}{|c|}{ Material Forrageiro } \\
\hline & & & & Caule Fino & Folha & Total \\
\hline $\mathrm{T} 1$ & $9.758,8$ & $2.253,0$ & $1.985,0$ & $1.898,1$ & $3.621,7$ & $5.519,8$ \\
\hline T2 & $12.269,4$ & $2.555,3$ & $2.561,4$ & $2.511,5$ & $4.641,2$ & $7.152,7$ \\
\hline T3 & $14.497,0$ & $3.080,9$ & $3.087,2$ & $3.071,2$ & $5.257,6$ & $8.328,8$ \\
\hline $\mathrm{T} 4$ & $13.819,8$ & $2.894,0$ & $3.034,5$ & $2.821,5$ & $5.069,9$ & $7.891,4$ \\
\hline
\end{tabular}


Tabela 5. Estimativa da quantidade de cinza existente nos tecidos das diferentes partes da planta $\left(\mathrm{kg} \mathrm{ha}^{-1}\right)$, de acordo com os tratamentos

\begin{tabular}{|c|c|c|c|c|c|c|}
\hline \multirow{3}{*}{ Tratamento } & \multicolumn{6}{|c|}{ Parte colhida } \\
\hline & \multirow{2}{*}{ Total } & \multirow{2}{*}{ Lenha } & \multirow{2}{*}{ C. Grosso } & \multicolumn{3}{|c|}{ Material Forrageiro } \\
\hline & & & & Caule Fino & Folha & Total \\
\hline $\mathrm{T} 1$ & $1.478,5$ & 67,6 & 70,7 & 141,1 & $1.199,2$ & $1.340,24$ \\
\hline T2 & $1.948,8$ & 78,4 & 105,9 & 211,2 & $1.553,41$ & $1.764,63$ \\
\hline T3 & $2.302,2$ & 106,3 & 136,4 & 328,6 & $1.730,81$ & $2.059,42$ \\
\hline T4 & $2.222,0$ & 90,9 & 119,6 & 253,4 & $1.758,23$ & $2.011,60$ \\
\hline
\end{tabular}

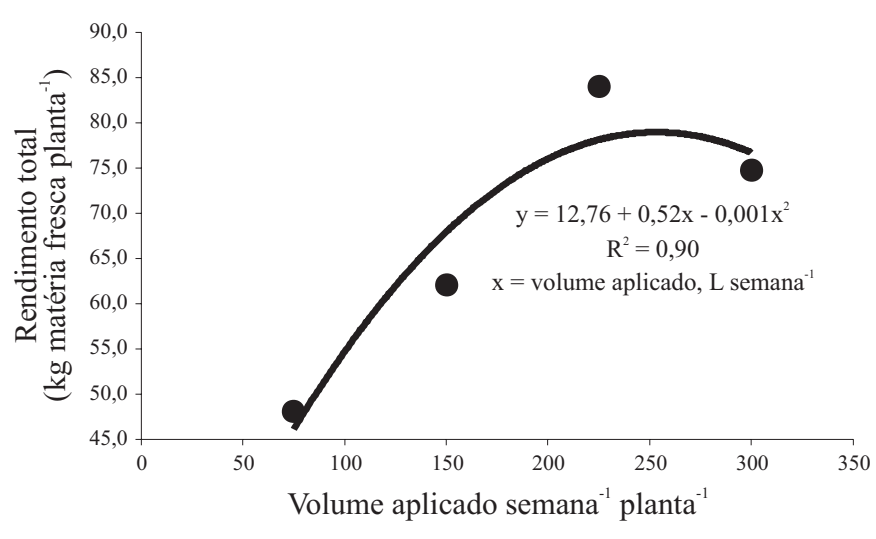

Figura 4. Regressão do volume de água aplicado como irrigação nos quatros tratamentos e os respectivos rendimentos de biomassa por planta

Considerando-se o total de água de irrigação aplicado e a salinidade média do efluente, que foi de $8,29 \mathrm{dS} \mathrm{m}^{-1}$ $\left(5,3 \mathrm{~g} \mathrm{~L}^{-1}\right)$ conclui-se que o tratamento $\mathrm{T} 1$ foi o mais eficiente na retirada de sais do solo, tendo em vista que o conteúdo de cinza é a estimativa aproximada da dessalinização do solo. Neste trabalho, o tratamento T1 retirou 13,84\% do total de sais que foram incorporados ao solo pela irrigação, enquanto o tratamento T4 retirou apenas 5,2\%.

\section{CONCLUSÕES}

1. A salinidade do solo aumentou em todos os tratamentos.

2. O melhor rendimento físico da erva-sal foi conseguido com a aplicação semanal de 225 litros de efluente da dessalinização de água salobra, após o mesmo ter servido como meio de cultivo para a tilápia rosa (Oriochromis sp.).

3. Para o tratamento T4, que recebeu semanalmente $300 \mathrm{~L}$ de efluente planta ${ }^{-1}$, o rendimento foi inferior ao que recebeu $225 \mathrm{~L}$, porém eles não são diferentes estatisticamente, mas os valores médios da condutividade elétrica foram menores e com diferenças pequenas entre as camadas do perfil que recebeu maior volume de irrigação.

4. O tratamento T1 apresentou o melhor rendimento de biomassa pelo total de água aplicada, chegando a produzir 4,84 g de matéria seca $\mathrm{L}^{-1}$ de efluente, enquanto o tratamento T4 produziu apenas $1,71 \mathrm{~g}$ de matéria seca $\mathrm{L}^{-1}$.

5. Considerando-se a incorporação de sais ao solo pela ir- rigação e sua retirada pela planta, o tratamento $\mathrm{T} 1$ foi o mais eficiente, com uma retirada de $13,84 \%$ do total incorporado, enquanto o percentual de retirada para o tratamento T4 foi de apenas 5,20\%.

\section{LITERATURA CITADA}

Amorim, M. C. C. de; Porto, E. R.; Silva Júnior, L. G de A.; Liberal, G. de S. Efeito de sais no solo provenientes de rejeitos da dessalinização por osmose inversa, no semi-árido pernambucano. In: Congresso Brasileiro de Engenharia Agrícola, 26., 1997, Campina Grande. Anais... Campina Grande: SBEA; UFPB, 1997. CD-Rom

Brown, J. J.; Glenn, E. P.; Fitzsimmons, K. M.; Smith, S. E. Halophytes for treatment of saline aquaculture efluent. Aquaculture, Amsterdam, v. 175, p. 255-268, 1999.

Claessem, M. E. C. (org.) Manual de métodos e análises de solo, 2. ed. rev. atual. Rio de Janeiro: EMBRAPA-CNPS, 1997. 212p. Documentos, 1

EMBRAPA. Empresa Brasileira de Pesquisa Agropecuária. Centro Nacional de Pesquisa de Solos (Rio de Janeiro, RJ). Sistema brasileiro de classificação de solos. Brasília: Embrapa-SPI, 1999. 412p.

Glenn, E.; Tanner, R.; Miyamoto, S.; Fitzsimmons, K.; Boyer, J. Water use, productivity and forage quality of the halophyte Atriplex nummularia grown on saline waste water in desert environment. Journal of Arid Enviroments, London, v.38, n. 9, p. 45-62, 1998.

Miyamoto, S. Water use and potential irrigation scheduling of halophytes. In.: Halophyte utilization in agriculture, Agadir, Morocco: Bari-Institute. 1993. p.99-146.

Miyamoto, S.; Glenn, E. P.; Olsen, M. W. Growth, water use and salt uptake of four halophytes irrigated with highly saline water. Journal of Arid Environments, London, v.32, n. 2, p.141-159, 1996.

Miyamoto, S.; Glenn, E. P.; Singh, N. T. Utilization of halophytic plants for fodder production with brackish water in subtropic deserts. In: Squires, V. R.; Ayoub, A. T. (ed) Halophyte as a resource for livestock and for rehabilitation of degraded lands. Amsterdam: Kluwer Academic, 1994. p.43-75.

Montenegro, A.; Montenegro, S.; Silva, V. P.; Marinho, I.; Silva, J. G.; Bezerra, R.; Barros, M. K. Uso múltiplo da água subterrânea nas áreas de domínio do Programa Xingó, com ênfase no desenvolvimento de halófitas. In.: Congresso Mundial de Águas Subterrâneas, 1, 2000, Fortaleza. Anais... Fortaleza: Associação Brasileira de Águas Subterrâneas, 2000. CD-Rom 
Moore, R. T.; White, R. S.; Calowell, M. M. Transpiration of Atriplex confertifolia and Eurotia lantana in relation to soil, plant and atmospheric moisture stress. Canadian Journal of Botany, Ottawa, v.50, p.1411-1418, 1972.

O’Leary, J. W. A critical analysis of the use of Atriplex species as crop plant for irrigation with highly saline water. In: Ahmad, R.; San Pietro, A. (ed.). Prospects for biosaline research. Karachi: Karachi University, 1986. p. 416-432.

O’Leary, J. W.; Glenn, E. P.; Watson, M. C. Agricultural production of halophytes irrigated with seawater. Plant and Soil, Dordrecht, v. 89, p. 311-321, 1985.

Oliveira, M. C. de. Leucena: suplemento protéico para a pecuária do semi-árido no período seco. Petrolina, PE: Embrapa Semi-Árido. 2000. 14p. Circular Técnica, 51
Porto, E. R.; Amorim, M. C. C. de; Silva Júnior, L. G. de A. Uso do rejeito da dessalinização de água salobra para irrigação da ervasal (Atriplex nummularia). Revista Brasileira de Engenharia Agrícola e Ambiental, Campina Grande, v.5, n.1, p.111-114, 2001.

Rhoades, J. D. Use of saline and brackish water for irrigation: implications and role in increasing food production, conserving water, sustaining irrigation and controlling soil and water degradation. In: International Executive Council Meeting, 49.; ICID Afroasian Regional Conference, 10.; International Workshop on the Use of Saline and Brackish Water for Irrigation: Implications for the Management of Irrigation, Drainage and Crops, 1998, Bali, Indonesia. Proceedings... Bali: ICID-CIID, 1998. p.261-304.

Silva, D. J.; Queiroz, A. C. Análise de alimentos (métodos químicos e biológicos). Viçosa: UFV, 3.ed., 1990. 165p.

SAS Institute, SAS/FSP user's guide: release 6.03 edition. Cary, 1989. 331p. 